\title{
Research on harmonic characteristics of electronic instrument transformer
}

\author{
Yang Chunyan ${ }^{1, a^{*}, \text { Hu Haoliang }}{ }^{1, b}$,Li Dengyun ${ }^{1, c}$, Xiong Qianzhu ${ }^{1}$, \\ Nie Qi ${ }^{1}$,Xu Zili ${ }^{1}$, Wang Zhongming ${ }^{2}$ \\ ${ }^{1}$ China Electric Power Research Institute, Wuhan, 430074, China \\ ${ }^{2}$ Hegang Power Supply Company,Hegang,154101,China

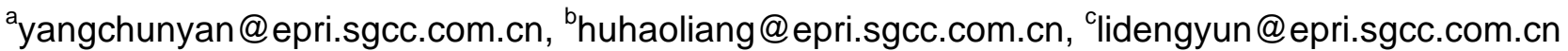

Keywords: Harmonic; electronic instrument transformer; Harmonic error measurement system Abstract. A harmonic error measurement system which can simulate the actual operating conditions of electronic instrument transformer was established for electronic instrument transformer harmonic error measurement. The system consists of High-power current and voltage harmonic source which is used to output fundamental and harmonic signal, harmonic voltage and current standard device and harmonic analyzer. The results indicate that after superposing the fundamental and harmonic, ratio and phase error of electronic current transformer (ECT) becomes larger with increasing frequency; ratio error of electronic voltage transformer(EVT) increases with the increasing harmonics frequency, but EVT phase error changed to negative value at rated current, $150 \mathrm{~Hz}$ frequency.

\section{Introduction}

With the power electronics technology widely used in power systems and distributed energy connected to the grid in succession, the harmonic pollution is increasingly serious, How real-time, accurately detect the harmonic power is getting more and more attention. The State Grid Corporation proposed that the strong intelligence network will be fully completed before 2020, Electronic instrument transformer is the decisive equipment for building digital substation and plays a vital role [1,2]. Current method for electronic instrument transformer harmonic characteristic research used in domestic is frequency characteristic method; it obtains the frequency characteristic by giving current (voltage) signals with different frequencies respectively in the primary side of electronic instrument transformer. However, this test method is different from the actual operation of electronic instrument transformer; there is a hidden danger for the safe operation of the power system.

In this paper, High-power current and voltage harmonic source, harmonic standard device and harmonic analyzer is researched. Harmonic source output the synthesized signal with fundamental and harmonic signal for simulating the harmonic wave of electronic instrument transformer in actual operating conditions. Electronic instrument transformer Harmonic error measurement of fills gaps in the field of harmonic measurement under actual operating conditions.

Electronic instrument transformer consists of electronic current transformer (ECT) and electronic voltage transformer (EVT). ECT includes optical current transformer, Rogowski coil current transformer and low power current transformer; EVT includes voltage transformer based on the linear electro-optic effect and voltage transformer based on the principle of capacitive voltage divider, inductive voltage divider and resistive and capacitive voltage divider. A low power electronic current transformer and an electronic voltage transformer based on the principle of inductive voltage divider were chosen as the sample for harmonic test.

\section{Harmonic Error Measurement System}

Harmonic error measurement system consists of harmonic generator, power amplifier, voltage booster, harmonic standard device and harmonic analyzer. 
Harmonic Generator The harmonic generator is equipped with microcomputer control; it is designed and manufactured by using digital waveform synthesis technology and modern power electronic technology, it has stable voltage output, high accuracy, wide frequency range, good repeatability, it can achieve 2th to 11th harmonics independent output and adjustable phase. The voltage waveform distortion of each harmonic is extremely low, and the fundamental frequency stability is better than $0.05 \%$, the frequency stability of each harmonic is better than $0.5 \%$, the phase control accuracy is up to $1^{\circ}$, the output voltage stability of inverter is better than $1 \%$ and load regulation is better than $1 \%$, the harmonic generator also has perfect overvoltage, overcurrent, overload and over-temperature protection.

The harmonic generator outputs $(0-350) \mathrm{V},(0-107) \mathrm{A}$, the total power is $30 \mathrm{kVA}$, the total harmonic output power is more than $20 \%$ of the total power, and the harmonic generator can simulate the harmonic voltage and current of electronic instrument transformer under the actual operating conditions, thereby creating the conditions for its calibration of frequency characteristic. Test current can reach $3000 \mathrm{~A}$ and test voltage can reach $500 \mathrm{kV}$ by using the harmonic generator and appropriate heat flow device, amplifier or voltage booster.

Harmonic Current Standard Device In order to choses the appropriate harmonic current standard device, a single-frequency current transformer standard experiment was conducted, and error characteristics comparison test at different frequency was conducted for existing current ratio standard and shunt in laboratory by using the method of electronic calibrator measuring error. The results showed that the influence of on the measurement results is small and can be ignored with respect to the effect of frequency, so the primary current is set to 50A for error test.

The shunt is chosen as the reference, and the comparison result of current transformers and shunt is shown in Fig. 1. Curve A represents result with $0.2 \Omega, 0.01$ level DC standard resistance as the sampling resistor of CT secondary side; Curve B represents result with $0.3 \Omega, 1 \%$ level non-inductive resistance as the sampling resistor of CT secondary side;Curve C represents result with $20 \Omega, 0.01$ level AC resistance as the sampling resistor of 100/1 current transformer connected to the CT secondary side. Fig. 1 shows that curve A has the maximum phase error change with frequency;curve B shows a low accuracy, thus resulting in a big ratio error; Curve $C$ shows a perfect result, the phase error change with frequency is similar to that of curve B. One reason for this result is that with non-inductive resistance did not have an indicated operating frequency range; the second reason is that inductance proportion of $20 \Omega$ resistor is relatively small.

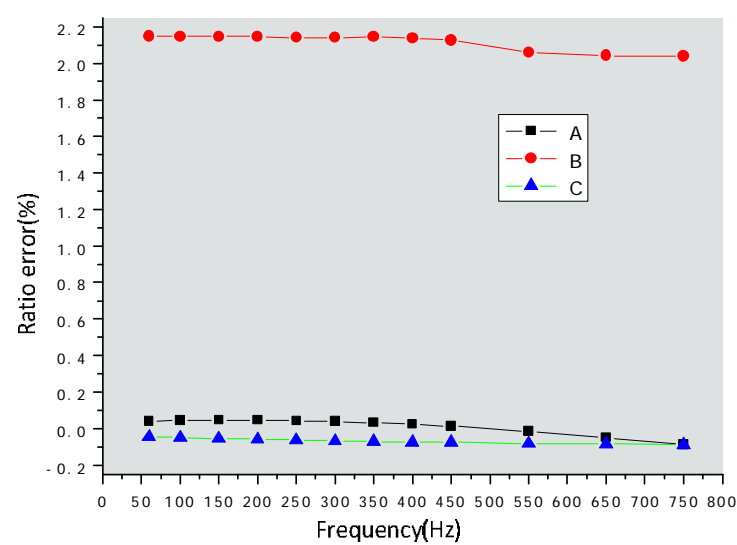

(a)Ratio error change with the frequency

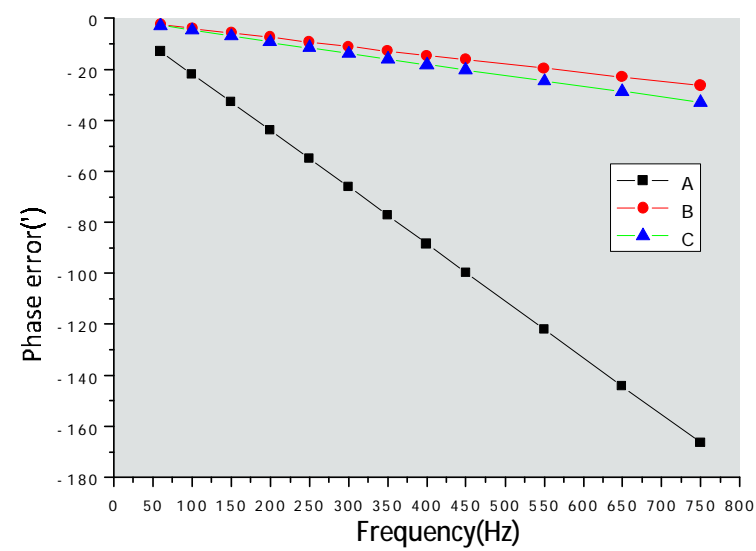

(b) Phase error change with the frequency

Fig.1 Comparison of current transformer and shunt

DC standard resistance and the AC resistance used in tests have a greater inductance at high frequency;the accuracy is not guaranteed and is not suitable for sampling resistor. Non-inductive resistance cannot also be chosen as the sampling resistor because of its low accuracy and unindicated operating frequency range. In order to solve such problem, a high-precision non-inductive resistor with wide frequency range is needed as the sampling resistor. 
Thus, a shunt with wide frequency range is chosen as the standard device for harmonic current. A Broadband coaxial shunt NORMA SHUNTS manufactured by FLUKE is chosen as the harmonic current standard device, Bandwidth is $(0-2) \mathrm{MHz}$, shunt Accuracy is $\pm 0.1 \%$, the phase shift is \pm $0.1 \% 100 \mathrm{kHz}$, temperature drift is less than $10 \mathrm{ppm} / \mathrm{K}$.

Harmonic Current Standard Device Electromagnetic transformer and capacitive voltage transformer is used in current power system for primary voltage measurements. The theoretical analysis and existing experimental studies have shown that frequency has great impact on the capacitive voltage transformer errors, so capacitive voltage transformer will not be chosen as the standard device for harmonic measurement $[3,4,5]$. Relevant literature in foreign had analyzed the frequency response characteristic of electromagnetic voltage transformer by experiments, the results indicate that amplitude error under $1 \mathrm{kHz}$ is less than $3 \%$, but it didn't obtain the phase frequency characteristic curve[6,7,8].

Theoretically, the resistor divider, capacitive voltage divider and resistive and capacitive divider has a wide frequency range, so the distributed capacitance has no effect on voltage division ratio of resistor divider in DC or low frequency, thus resistor divider is widely used in low frequency high voltage measurement technology. Capacitive voltage divider use the capacitance of dividing capacitor and sampling capacitor at the AC signal for voltage dividing, so it is suitable for AC signal dividing, and it has a good high-frequency performance. Resistive and capacitive divider use RC series network for voltage dividing, and has a good high-frequency and low-frequency performance; the disadvantage is complex debugging for high-impedance and low-capacitance network.

So the capacitive voltage divider and resistive and capacitive divider is suitable as the standard device and is used for amplitude-frequency characteristic test in (50 2000)Hz.

Capacitive voltage divider is chosen as the harmonic voltage standard device, and $0.005 \%$ level standard PT as the calibration device, calibration results are shown in Fig.2, the ratio error is less than $0.005 \%$ and phase error is less than $0.3^{\prime}$.

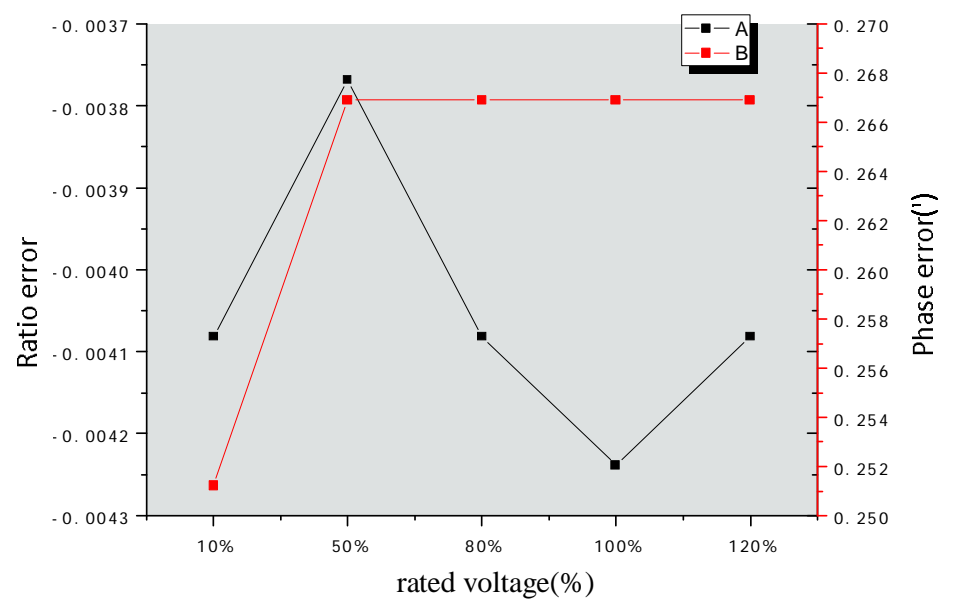

Fig.2 Calibration of capacitive voltage divider

Electronic Instrument Transformer Harmonic Analyzer Electronic instrument transformer harmonic analyzer uses portable computer as a whole harmonic analysis platform, the system consists of 24bits high-precision dual-channel synchronous acquisition card and virtual instrument technology. Acquisition system collects input voltage signal by high-precision acquisition card. Combining with high-precision FFT correction algorithms $[9,10,11]$ and the internal clock synchronization technology, it can be ensured strictly two channel sample synchronously. The indicators of system are as follows: Input voltage range (RMS 3.5V, 700mV); Sampling rate of 50kHz-15MHz, Resolution of 24 bits; accuracy of $0.05 \%$. The high sampling rate and measurement accuracy shows that the electronic Instrument Transformer calibrator can be used as calibration device for harmonic measurement test. 


\section{Electronic Instrument Transformer Error Test}

ECT Harmonic Test The test circuit principle of ECT harmonic accuracy is shown in Fig.3. Harmonic current generator generates the current signal superimposed with fundamental current and each harmonic current, the superimposed current is amplified through power amplifier, the tested ECT and traceable ECT are connected in series with the coaxial shunt for spectrum analysis and deviation Analysis. When giving pure fundamental signal, record the average value of measurement error at some percentage of the rated current; When giving the pure fundamental signal, 3rd harmonic wave (about $5 \%$ of fundamental signal), 5th harmonic wave (about $4 \%$ of fundamental signal) and 7 th harmonic wave (about $3 \%$ of fundamental signal), then record the average value of measurement error at some percentage of the rated current;

A $110 \mathrm{kV}$ low-power ECT (Rated current 1600A) and 10kV low-power ECT (Rated current $600 \mathrm{~A})$ are chosen as the tested ECT.

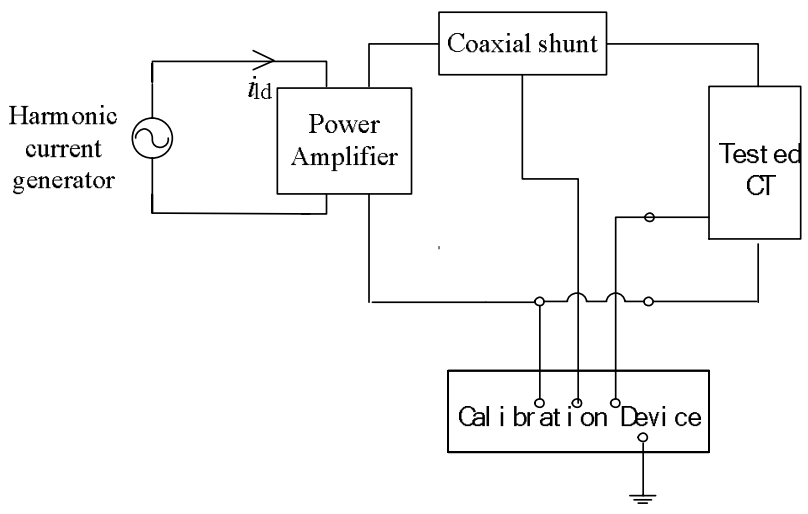

Fig. 3 test circuit principle of ECT harmonic accuracy

EVT Harmonic Test The test circuit principle of EVT harmonic accuracy is shown in Fig.4.

Harmonic voltage generator generates the voltage signal superimposed with rated fundamental voltage and each harmonic voltage, the superimposed voltage is amplified through boosting transformer T2, the tested EVT and traceable capacitive voltage divider are connected to output terminal in parallel for spectrum analysis and deviation Analysis. When giving pure fundamental signal, record the average value of measurement error at some percentage of the rated voltage; When giving the pure fundamental signal, 3rd harmonic wave (about $5 \%$ of fundamental signal), 5 th harmonic wave (about $4 \%$ of fundamental signal) and 7 th harmonic wave (about 3\% of fundamental signal), then record the average value of measurement error at some percentage of the rated voltage;

A $10 \mathrm{kV}$ Inductance division type EVT (Rated voltage $10 \mathrm{kV} / \sqrt{3}$ ) is chosen as the tested ECT. Single-phase

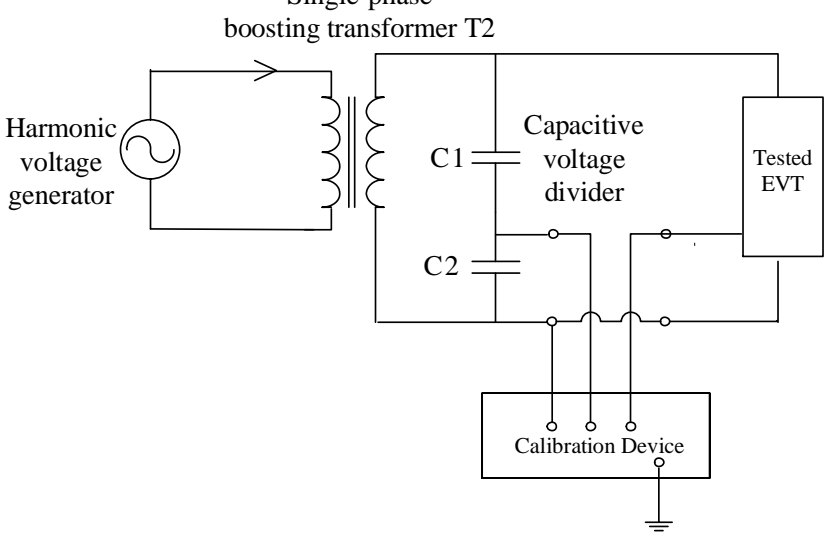

Fig.4 test circuit principle of EVT harmonic accuracy 


\section{Result Analysis of Harmonic Error Test}

$110 \mathrm{kV}$ ECT harmonic characteristic $110 \mathrm{kV}$ ECT harmonic characteristic is shown in Fig.5, where Fig.5 (a) shows the error of fundamental wave, Fig.5 (b), (c) shows ratio and phase error change with harmonic wave. Fig.5 indicates that ratio and phase error of ECT fundamental wave did not change substantially when superimposing fundamental and harmonics wave. In the case of superimposing and harmonic wave, ECT ratio error had less volatile under each harmonic wave and the absolute values of ratio error increases with the increasing harmonics frequency, the phase error had the similar changes.

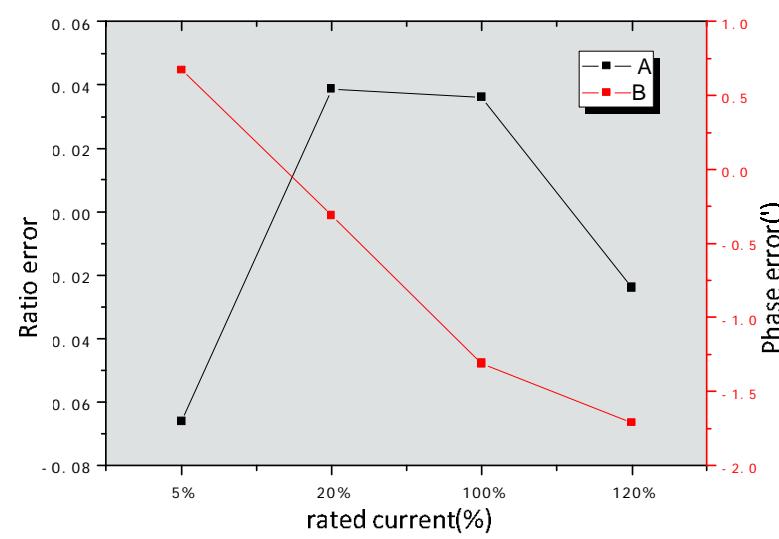

(a)The error of fundamental wave

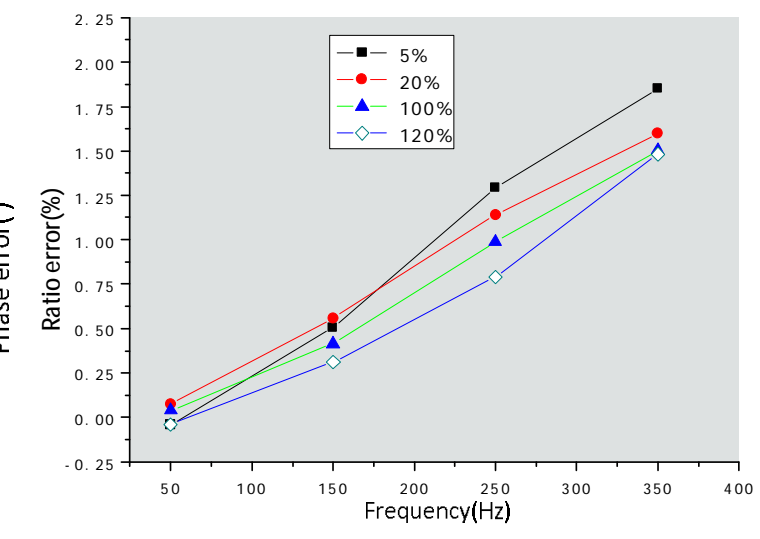

(b)Ratio error change with harmonic wave

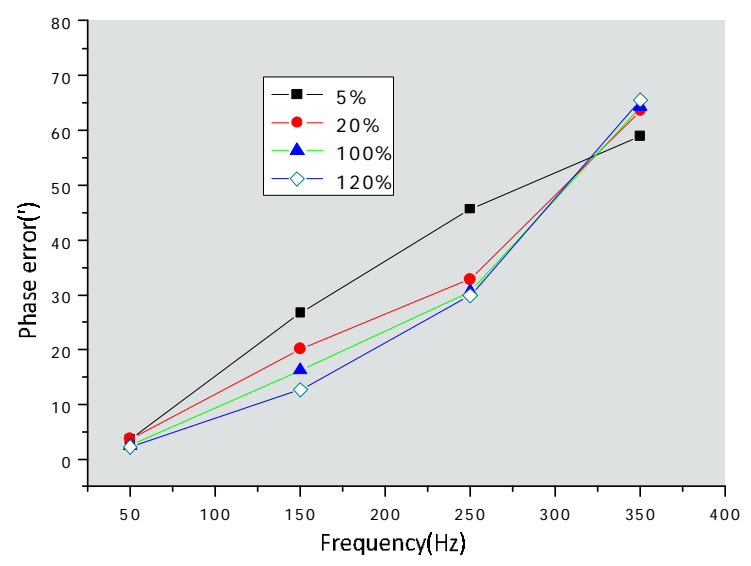

(c) Phase error change with harmonic wave

Fig.5 $110 \mathrm{kV}$ ECT harmonic characteristic 
10kV EVT harmonic characteristic 10kV EVT harmonic characteristic is shown in Fig.6, where Fig.6 (a) shows the error of fundamental wave, Fig.6 (b), (c) shows ratio and phase error change with harmonic wave.

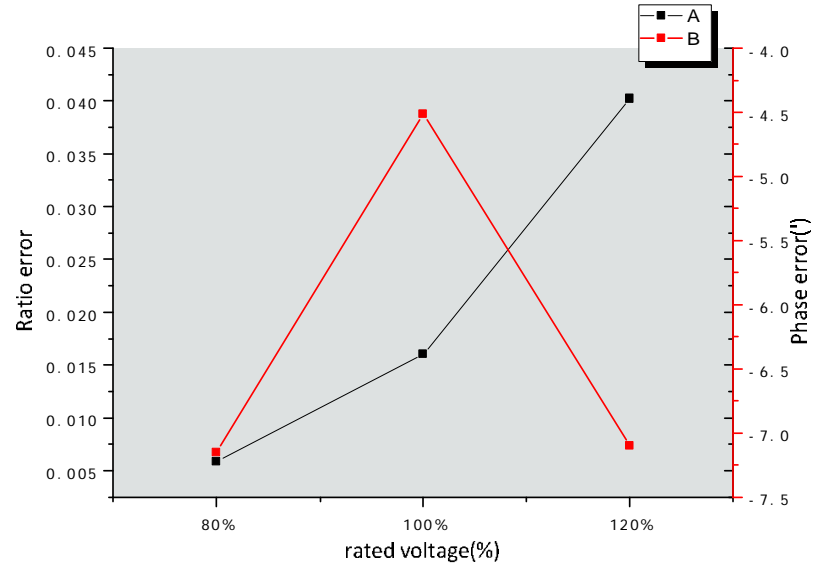

(a) The error of fundamental wave

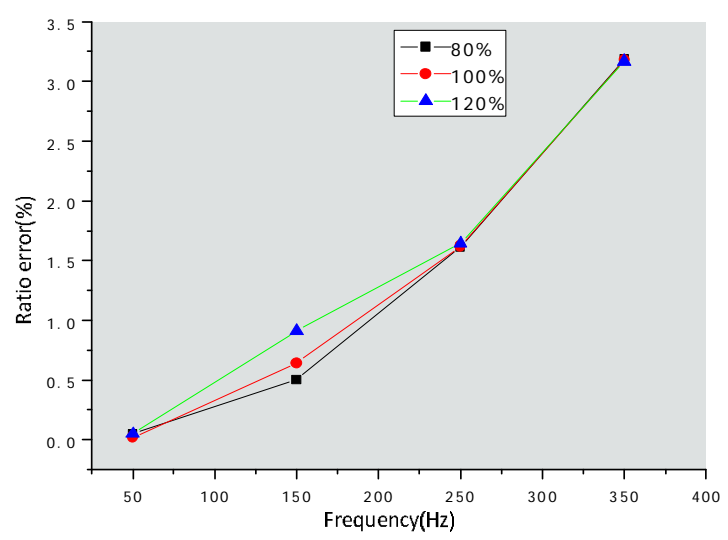

(b) Ratio error change with harmonic wave

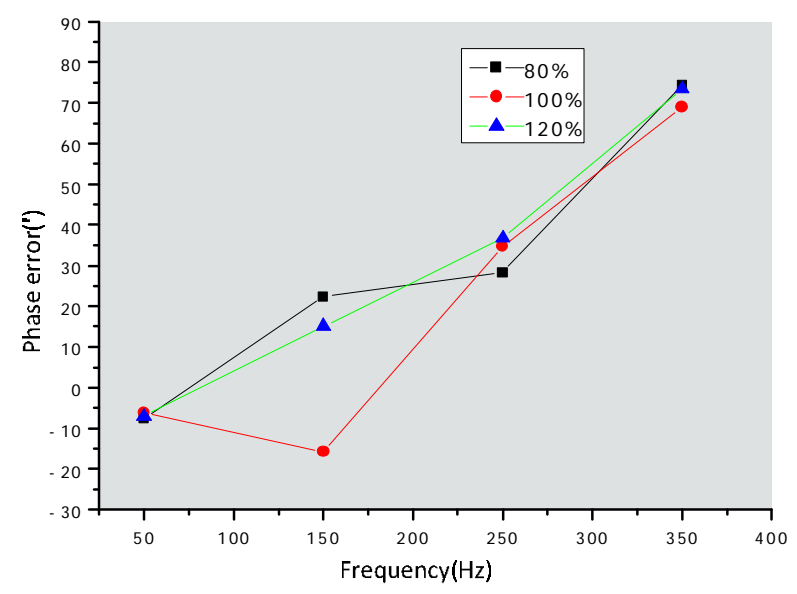

(c) Phase error change with harmonic wave

Fig.6 $10 \mathrm{kV}$ EVT harmonic characteristic

Fig. 6 indicates that ratio and phase error of EVT fundamental wave did not change substantially when superimposing fundamental and harmonics wave. In the case of superimposing harmonic wave, EVT ratio error had less volatile under each harmonic wave and the absolute values of ratio error increases with the increasing harmonics frequency; EVT phase error had large volatile under each harmonic wave and changed to negative value at rated current, $150 \mathrm{~Hz}$ frequency, the overall trend is not obvious, and the specific reason needs further experimental analysis.

\section{Conclusion}

An Electronic Instrument Transformer harmonic error measurement system was established in this paper, and a harmonic test for $110 \mathrm{kV}$ ECT and $10 \mathrm{kV}$ EVT is conducted in such system, the results indicated that ratio and phase error of ECT fundamental wave did not change substantially when superimposing fundamental and harmonics wave, In the case of superimposing and harmonic wave, ECT ratio error and phase error increases with the increasing harmonics frequency, and EVT ratio error increases with the increasing harmonics frequency, but EVT phase error changed to negative value at rated current, $150 \mathrm{~Hz}$ frequency. 


\section{References}

[1] LI Qian, ZHANG Shu-han, LU Shu-feng, et al. Comparison of Two Electronic Transformer Error Measuring Methods [J]. High Voltage Engineering, 2011, 12(37):3022-2027.

[2] TAN Hong-en, HU Hao-liang, LEI Min, et al. Experimental Analysis of On-site Calibration of Electronic Instrument Transformer [J]. High Voltage Engineering, 2010, 12(36): 2990-2995.

[3] Yao Xiao, Jun Fu, Bin $\mathrm{Hu}$, et al. Problems of voltage transducer in harmonic measurement [J]. IEEE Transactions on Power Delivery, 2004(3):1483-1487.

[4] Li Pengfei, Zhang Jiwei, Zhou Xichao. Harmonic Voltage Measurement and Analysis of Capacitor Voltage Transformer. Guansu Electric Power Technology, 2010,4:49-50.

[5] F.Ghassemi, P.Gale, T.Cumming, et al. Harmonic Voltage measurements Using CVTS [J]. IEEE Transactions on Power Delivery, 2005, 1 (20):443-449.

[6] D.A.Douglass. Potential Transformer Accuracy at $60 \mathrm{~Hz}$ voltages above and below rating and at frequencies above $60 \mathrm{~Hz}$ [J].IEEE Transactions on Power Apparatus and Systems, 1981, 3(PAS-100):1370-1375.

[7] Luszcz.J, Moson.I. Modelling of voltage transformer performance in power system harmonic frequency range, Nonsinusoidal Currents and Compensation, 2008.

[8] Peter E. Harmonic measurements in Industrial Power Systems [J], IEEE Transactions on Industry Applications, 1995, 1(31):175-183.

[9] Liu Xudong. Harmonic Detection Algorithm and Testing System Research in Power System [D]. Shenyang University of Technology, 2009.

[10] Wang Jun. The Research of Harmonic Measurement Method Based on FAFT Algorithm [D]. Northwest University, 2004.

[11] Sachin K. Jain, S.N. Singh. Harmonics estimation in emerging power system: Key issues and challenges [J]. Electric Power Systems Research, 2011, (81): 1754- 1766. 